\title{
Compatibility of detached divertor operation with robust edge pedestal
}

\section{performance}

\author{
A.W. Leonard ${ }^{\mathrm{a}}$, M.A. Makowski ${ }^{\mathrm{b}}$, A.G. McLean ${ }^{\mathrm{b}}$, T.H. Osborne ${ }^{\mathrm{a}}$, and P.B. Snyder ${ }^{\mathrm{a}}$ \\ ${ }^{a}$ General Atomics, PO Box 85608, San Diego, California 92186-5608, USA \\ ${ }^{b}$ Lawrence Livermore National Laboratory, Livermore, California, USA
}

\begin{abstract}
The compatibility of detached radiative divertor operation with a robust $\mathrm{H}$-mode pedestal is examined in DIII-D. A density scan produced low temperature plasmas at the divertor target, $\mathrm{Te} \leq 2 \mathrm{eV}$, with high radiation leading to a factor of $\geq 4$ drop in peak divertor heat flux. The cold radiative plasma was confined to the divertor and did not extend across the separatrix in X-point region. A robust $\mathrm{H}$-mode pedestal was maintained with a small degradation in pedestal pressure at the highest densities. The response of the pedestal pressure to increasing density is reproduced by the EPED pedestal model. However, agreement of the EPED model with experiment at high density requires an assumption of reduced diamagnetic stabilization of edge Peeling-Ballooning modes.
\end{abstract}

Abstract length (words): 120 currently

PACS: 52.55.Fa, 52.55.Rk, and 52.55.Tn

PSI-20 Keywords: DIII-D, Divertor Detachment, Pedestal

${ }^{*}$ Corresponding and presenting author address: A.W. Leonard, c/o General Atomics, PO Box 85608, San Diego, California 92186-5608, USA.

Corresponding and presenting author e-mail: leonard@fusion.gat.com 


\section{Introduction}

Maintaining tolerable heat loads to plasma facing surfaces (PFCs) in future high power density magnetic fusion devices will require dissipation of a large fraction of the exhaust power through radiation and other atomic physics processes. Such dissipation is achieved in current tokamaks through gas puffing of additional fueling gas to raise the divertor plasma density and lower its temperature for increased radiation from intrinsic and injected impurities, neutral particle interactions and plasma recombination. A divertor plasma state that exhibits a large reduction of exhaust power, plasma pressure and ion flux at the divertor target is generally referred to as a detached divertor plasma. However, detached divertor operation has also been associated with reduced energy confinement in the core plasma, calling into question its compatibility with a high performance core plasma in future reactor scale tokamaks [1-3]. The core performance degradation associated with detached divertor operation is usually due to a reduction in the H-mode edge pedestal pressure and resulting lower core confinement associated with stiff core profiles. The lower edge pedestal pressure with divertor detachment may be a result of higher losses due to radiation or high neutral fluxes inside the separatrix, or alternatively due to a reduction in the pedestal MHD stability limit at higher collisionality and associated reduction in edge current. Understanding the mechanism of pedestal degradation with detached divertor operation in existing devices is necessary for scaling the pedestal performance to reactor scale tokamaks with a low collisionality pedestal even for detached radiative divertor operation. Further, improved understanding of the interaction between the pedestal and detached divertor operation will be necessary for the development of divertor heat flux control in future devices [4-5].

In this study a density scan in DIII-D H-mode discharges is examined to determine the dependence of the edge pedestal pressure on divertor conditions. In DIII-D, detached radiative divertor plasmas are produced by deuterium-only injection where the gas puffing raises the 
divertor density and lowers the electron temperature for efficient radiation from the intrinsic carbon impurity, $\sim 2 \%$. Typically carbon provides $2 / 3$ of the radiation while hydrogenic emission provides the remainder [6]. The intense radiation and plasma-neutral interaction results in a "detached" divertor with cold dense divertor plasma, $\mathrm{T}_{\mathrm{e}} \leq 2 \mathrm{eV}$, where plasma pressure and heat flux at the target are greatly reduced from that at the entrance to the divertor region. The DIII-D detached divertor plasmas have similar characteristics to those in metal wall tokamaks where injected low-Z impurities, typically nitrogen or neon, provide the additional radiation $[7,8]$. In general the $\mathrm{H}$-mode pedestal pressure dependence on density is found to be consistent with the EPED model [9] of pedestal stability and transport, indicating no inherent interaction with the detached divertor plasma causing further degradation of the H-mode pedestal. However, at the highest collisionalities further refinement of the pedestal model may be required to fully describe the observations. In Sec. 2 the experimental observations from the density scan are presented. This includes characterization of the divertor plasma as well as the pedestal. In Sec. 3 the pedestal pressure dependence on density is compared to the MHD stability limit and EPED model. In Sec. 4 the implications of these results for reactor scale tokamaks are discussed.

\section{Density Scan Experimental Observations}

Seven discharges of a density scan in edge localized mode (ELM)ing H-mode, Fig. 1, were run at 1.1 MA, 1.95 $\mathrm{T}$ for $\mathrm{q}_{95}$ of 4.3 with 5.4 MW of neutral beam injection (NBI) auxiliary heating. The shaping was modest with an upper triangularity of $\delta_{\mathrm{u}} \sim 0.2$ and a higher lower triangularity of $\delta_{1} \sim 0.5$. The line-averaged density was varied from $5.0 \times 10^{19} \mathrm{~m}^{-3}$ to $9.0 \times 10^{19} \mathrm{~m}^{-3}$, or normalized density $\mathrm{n}_{\mathrm{e}} / \mathrm{n}_{\mathrm{GW}} \sim 0.5-0.9$, by a deuterium fueling rate from the outboard main chamber ranging from $4 \mathrm{~Pa}-\mathrm{m}^{3} / \mathrm{s}$ up to $40 \mathrm{~Pa}-\mathrm{m}^{3} / \mathrm{s}$. The fueling scan resulted in increasing divertor density and radiation and decreasing divertor target electron temperature and heat flux. The core plasma carbon fraction was $\sim 2 \%$, or $Z_{\mathrm{eff}}=1.6$, for the low density discharges which 
decreased to $Z_{\mathrm{eff}}=1.4$ for the highest density. The pedestal top density covered a range of $3.8 \times 10^{19} \mathrm{~m}^{-3}$ to $7.1 \times 10^{19} \mathrm{~m}^{-3}$ due to the increase in external fueling. The global stored energy, $\beta_{\mathrm{N}} \sim 2.2$ remained constant over the density scan, though due to its density dependence the normalized confinement factor, $\mathrm{H}_{98 \mathrm{Y} 2}$ dropped from 1.35 to 1.15 at the highest density, At the highest density an additional discharge was run with slightly lower auxiliary heating, 4.6 MW, which resulted in significantly lower pedestal pressure and stored energy, $\sim 25 \%$. The reasons for this reduction are described in Sec. 3 on pedestal analysis.

The divertor conditions in the density scan were measured with divertor Thomson scattering (DTS) to determine the onset of divertor detachment, as shown in Fig. 2. While the details of the divertor plasmas of this scan are described in Ref [10], a brief description of relevant details is also given here. Divertor detachment occurs when dissipation of energy and pressure reduces the target plate heat and particle flux below that expected from the simple 2-point SOL model [11]. While the Degree of Detachment (DOD) metric [12] from Langmuir probe ion flux unavailable, a more direct observation of divertor detachment can be seen in the DTS and power balance measurements of Fig. 2. The electron temperature near the outboard divertor target steadily decreases with increasing density, Fig. 2(a), until over a narrow range of pedestal density at $\sim 6 \times 10^{19} \mathrm{~cm}^{-3}, \mathrm{~T}_{\mathrm{e}}$ drops sharply from above $\sim 15 \mathrm{eV}$ to below $2 \mathrm{eV}$. This low temperature region is indicative of divertor detachment where ion-neutral collisions dominate over ionization resulting in a plasma pressure reduction of a factor of 2-4 compared to the attached cases. The drop in divertor temperature is accompanied by an large increase in outboard divertor density, and radiation, largely dissipating the exhaust power as shown in Fig. 2(b). The peak heat flux between ELMs is reduced a factor of $\sim 4$ with the remaining heat flux under detached divertor conditions, primarily a result of radiative heating of the target $[13,14]$.

A further examination of the detached divertor conditions is shown in Fig. 3 for the highest density case. The $2 \mathrm{D}$ profile of the divertor electron temperature is produced by sweeping the 
divertor configuration across DTS measurement locations and removing any data points during an ELM pulse [3]. The 2D profile shows a low temperature, $\mathrm{T}_{\mathrm{e}} \leq 5 \mathrm{eV}$ across the target plate extending from the separatrix far into the SOL. This condition is typically referred to as "complete" detachment. The low temperature region also extends above the target plate encompassing the bulk of the divertor plasma, but does not, however, extend across the separatrix into the core plasma. Inversion of bolometry data for the $2 \mathrm{D}$ radiated power profile exhibits a similar pattern. The strong radiation region extends throughout the divertor, but does not extend inside the separatrix into the pedestal. The plasma profiles of Fig. 3 and bolometric inversions indicate the cold dense radiating plasma, or MARFE, is excluded from the core plasma inside the separatrix avoiding anomalous loss of power from the pedestal.

The H-mode pedestal profile response to the density scan is shown in Fig. 4. The pedestal electron density and temperature profiles were produced by fitting the midplane edge TS data collected in the last half of the cycle from one ELM to the next, during constant H-mode conditions. The ion temperature is obtained from charge-exchange-recombination (CER) measurements of CVI ions, also collected in the last half of the ELM cycle. The total pressure profile also accounts for deuterium ion dilution obtained from CVI ion density CER measurements. The fueling scan produced an evenly distributed, slightly less than factor of 2 variation of pedestal density. The increasing density produced a commensurate reduction in the pedestal electron and ion temperature. The resulting total pressure profile, [Fig. 4(a)] remained approximately constant from low density to moderately high density. At the highest density with full auxiliary heating the pedestal pressure exhibited modest degradation. The lowest pedestal pressure profile of [Fig. 4(a)] was for the lower power case at the highest density. For this case, the pedestal pressure degradation was more severe, primarily through a reduction in the pedestal electron temperature. In the next section this dependence of the pedestal pressure on density is examined in the context of pedestal stability and transport. 


\section{Pedestal Stability and Transport}

The compatibility of detached divertor operation is examined in the context of the H-mode pedestal profiles that should be expected from the EPED model of pedestal stability and transport [9]. This model of the edge pedestal in the Type I ELMing regime is based on the assumption that the local pressure gradient is set by the kinetic ballooning mode (KBM) limit while the pedestal pressure height at ELM onset is set by the peeling-ballooning (PB) MHD stability limit, a more global limit taking into account the entire pedestal profile. Taken together, these two constraints predict the pedestal pressure height and width. The first assumption of this model is operation in Type I ELMing regime. This assumption applies to this entire dataset, with large and distinct ELMs through the density scan. The ELM frequency was $100-150 \mathrm{~Hz}$ for the lowest density case and represents the natural ELM frequency for these conditions. At the highest density the ELM frequency increased to $\sim 160 \mathrm{~Hz}$ with constant power and only a modest decrease in pedestal pressure. These discharges avoided the Type III ELM regime $[15,16]$, which can occur at high density with input power near the L-H transition power and typically results in a several fold increase in ELM frequency and $\geq 50 \%$ reduction in pedestal pressure. For the two highest density cases one was run with auxiliary heating of 4.6 MW compared to 5.4 MW for the rest of the density scan. The lower power resulted in a lower ELM frequency, $\sim 130 \mathrm{~Hz}$, characteristic of the Type I ELM regime, with a significantly lower pedestal pressure and global stored energy. These characteristics of the lower power discharge will be informative when examining different aspects of the pedestal model.

The MHD stability limit to the PB mode is first examined and found to be consistent with the measured pedestal profiles. The PB stability limit is examined by constructing magnetic equilibrium with measured plasma pressure profiles and a model of the edge current profile [17]. With the edge pressure profile obtained from TS and CVI CER measurements as 
described in the previous section, the edge current profile is assumed to follow the Sauter [18] bootstrap current model using the measured edge profiles. A lower level of current inferred from transport analysis of the ohmic current profile also adds to the current profile. Once the magnetic equilibrium is reconstructed with these constraints the MHD stability of the edge pedestal is analyzed with the ELITE code [19].

The resulting stability space from the analysis described above is shown in Fig. 5. It is seen to be consistent with the measured pressure gradient and modeled current profile to within experimental error. The stability curve shown by the solid line was calculated for the lowest density case and indicates the location where the growth rate of the PB mode becomes equal to $5 \%$ of the Alfven frequency. An alternative shown by the dashed line, assumes the critical growth rate is half of the effective diamagnetic frequency, $\omega *$ eff. These critical growth rate specifications for the PB mode have been motivated by two-fluid BOUT++ simulations [9]. Both stability criteria are within experimental uncertainty and exhibit several features of operation at higher density and collisionality. The horizontal part of the stability curve indicates the peeling boundary, where ELMs are driven by the edge bootstrap current. At higher density and collisionality the bootstrap current is suppressed and the edge pressure gradient becomes the limiting parameter. At the highest collisionality the limiting pressure gradient begins to decrease as the most unstable mode moves to higher toroidal mode number, $\mathrm{n}$, with less diamagnetic stabilization [9]. The measured maximum pedestal gradient for each density is marked by the symbols and exhibits a similar trend to the stability curve. With increasing collisionality and suppressed edge bootstrap current, the measured profiles exhibit a continuously decreasing pressure gradient, with an absolute magnitude very similar to the calculated limit. In order to allow discharges with different pedestal widths to be examined within the same stability diagram, the pedestal pressure gradient and current axes are normalized by one-quarter power of the pedestal width, $w^{1 / 4}$. This normalization is motivated 
by previous stability studies indicating the limiting pressure and current gradient is proportional to $w^{-1 / 4}[20]$. The highest density case departs from the stability curve, particularly that of the diagmagnetic stability criterium, because of the lower injected power, as mentioned earlier. This results in a drop of global beta from $\beta_{\mathrm{N}}=2.2$ to $\beta_{\mathrm{N}}=1.7$ with a smaller Shafranov shift and lower outboard midplane poloidal field for a lower pedestal pressure gradient limit. Overall the measured pressure gradients are in good agreement, in both magnitude and trend, with the calculated limits.

Combining the MHD stability limit described above with the assumption that the KBM limits the local pressure gradient, one may now predict the pressure at the top of the pedestal. The EPED1.63 model [9] embodies these constraints and is used to compare the pedestal heights in the model prediction with the experimental observations in this study, as shown in Fig. 6. The inputs to EPED1.63 are eight scalar parameters to describe the equilibrium: $B_{t}, I_{p}$, $\mathrm{R}, \mathrm{a}, \delta, \kappa, \beta_{\mathrm{N}}$ and $\mathrm{n}_{\mathrm{e}, \mathrm{ped}}$, where $\mathrm{R}$ is the geometric major radius, a is the minor radius, $\delta$ is the average triangularity, $\kappa$ is the elongation, $\beta_{\mathrm{N}}$ is the normalized global stored energy. Note that only the pedestal top density, $\mathrm{n}_{\mathrm{e}, \mathrm{ped}}$, is used to describe the state of the edge and divertor plasma conditions. The two criteria for critical growth rate of the ELM instability within this model are shown in Fig. 6. Differences between the two models become significant at high collisionality where diagmagnetic stabilization becomes more significant. Both models assume the same limit for the local pressure gradient as set by the onset of the KBM limit.

From low density to moderately high density both the diamagnetic and Alfven stabilization models predict the same pedestal height, remaining constant with density, but $\approx 10 \%$ higher than the measured pedestal pressure. This is well within experimental certainty given that the model is based on model profiles and model plasma shapes. Above a pedestal density of $\mathrm{n}_{\mathrm{e}, \mathrm{ped}}=$ $6 \times 10^{19} \mathrm{~m}^{-3}$, the two models diverge with the Alfven stabilization model better following the trend of the data, as also seen in Fig. 5. The diamagnetic stabilization model, however, moves 
to slightly higher pedestal pressure. This is due to the significant increase in diamagnetic frequency at high density stabilizing the PB mode. The data is clearly better correlated with the lower stabilization model with the growth rate normalized by the Alfven frequency. This model captures the modest drop in pedestal pressure at the highest density and, reproduces the dependence on global $\beta$ for the lower power case. Further work with two-fluid simulation would be helpful to examine the stabilization criteria.

\section{Discussion}

The compatibility of divertor heat flux control through detached divertor operation with a robust H-mode pedestal and high core plasma confinement has been examined in DIII-D. A density scan has documented dense, cold divertor target conditions, $\mathrm{T}_{\mathrm{e}} \leq 2 \mathrm{eV}, \mathrm{n}_{\mathrm{e}} \geq 40 \times 10^{19} \mathrm{~m}^{-3}$, with direct TS measurements. These conditions promote divertor heat flux dissipation through radiation and plasma recombination. The cold divertor plasma did not extend across the separatrix in the X-point region, allowing maintenance of a robust H-mode pedestal and good H-mode confinement. These discharges have demonstrated that heat flux dissipation through detached divertor operation can be compatible with high performance core plasma operation.

The dependence of the pedestal pressure on density and collisionality was examined in the context of the EPED pedestal model. The EPED1.63 model reproduced many of the features of the experimental observations including a constant pressure from low to moderately high density. At the highest densities a modest degradation of pedestal pressure was reproduced with an assumption of an Alfven stabilization criterium. This is an issue that would benefit from further simulation studies using two fluid tokamak models of MHD stability in realistic geometry. However, future large tokamaks, such as ITER and DEMO, are expected to operate with a lower collisionality pedestal with a robust stabilizing edge bootstrap current, even for highly dissipative detached divertor conditions. This lends further confidence that divertor heat 
flux control can be obtained while maintaining a robust pedestal and high performance core plasma in future burning plasma tokamaks.

\section{Acknowledgment}

This material is based upon work supported by the U.S. Department of Energy, Office of Science, Office of Fusion Energy Sciences, using the DIII-D National Fusion Facility, a DOE

Office of Science user facility, under Awards DE-FC02-04ER54698 and DE-AC52-07NA27344. DIII-D data shown in this paper can be obtained in digital format by following the links at https://fusion.gat.com/global/D3D_DMP 


\section{References}

[1] T.W. Petrie, et al. Nucl. Fusion 37 (1997) 321.

[2] M. Kotschenreuther, et al. Phys. Plasmas 14 (2007) 072502.

[3] A.W. Leonard, et al. Nucl. Fusion 52 (2012) 063015.

[4] A. Kallenbach, et. al., Plasma Phys. Control. Fusion 52 (2010) 055002.

[5] E. Kolemen, et al., This publication.

[6] R.C. Isler, et al., Phys. Plasmas 4 (1997) 355

[7] C. Giroud, et al., Nucl. Fusion 53 (2013) 113025

[8] M.L. Reinke, et al., Jour. Nucl. Mater. 415 (2011) S340

[9] P.B. Snyder, et al. Nucl. Fusion 51 (2011) 103016.

[10] A.G. McLean, et. al., This publication

[11] P.C. Stangeby, "The plasma boundary of magnetic fusion devices", Institute of Physics Publishing, Bristol, 2000

[12] A. Loarte, et al., Nucl Fusion 38 (1998) 331

[13] A.W. Leonard, et al., Phys. Rev. Lett. 78 (1997) 4769.

[14] A.W. Leonard, et al. Phys. Plasmas 5 (1998) 1736.

[15] E.J. Doyle, et al., Phys. Fluids B 3 (1991) 2300

[16] R. Sartori, et al., Plasma Phys. Control. Fusion 46 (2004) 723.

[17] A.W. Leonard, et al. Phys. Plasmas 15 (2008) 056114.

[18] O. Sauter, C. Angioni, Y.R. Lin-Liu, Phys. Plasmas 6 (1999) 2834.

[19] P.B. Snyder, et al. Nucl. Fusion 44 (2004) 320.

[20] P.B. Snyder, et al. Plasma Phys. Control. Fusion 46 (2004) A131. 


\section{List of Figure Captions}

Fig. 1. Density scan in DIII-D (a) Plasma current and auxiliary NBI heating, (b) line averaged density and (c) external deuterium fueling.

Fig. 2. As a function of pedestal density, (a) the divertor target $T_{e}$ and $n_{e}$ measured by Thomson scattering, and (b) the outboard divertor radiated power and peak surface heat flux.

Fig. 3. The $2 \mathrm{D}$ profile of the divertor and $\mathrm{X}$-point region electron temperature produced by sweeping the divertor configuration across the TS measurement locations. The small squares mark the measurement locations with the colored contour profile fitted to the measurements.

Fig. 4. Pedestal profiles obtained in the last half of the ELM phase for (a) total pressure, (b) electron density, (c) electron temperature and (d) CVI ion temperature. The three highest density cases are marked with dashed traces to highlight them.

Fig. 5. Stability diagram for the edge pedestal current and pressure gradient. The stability space is calculated for the magnetic equilibrium of the $\mathrm{n}_{\mathrm{e}, \mathrm{ped}}=3.8 \times 10^{19} \mathrm{~m}^{-3}$ discharge. The pressure gradient and current axes are normalized by the pedestal width, $w^{1 / 4}$ to allow display of the density scan discharges on the same diagram.

Fig. 6. The measured pedestal top pressure as a function of pedestal density. The EPED1.63 predictions for pedestal pressure for assumptions of full diamagnetic stabilization $(\times)$ and reduced diamagnetic stabilization $(\bullet)$. 


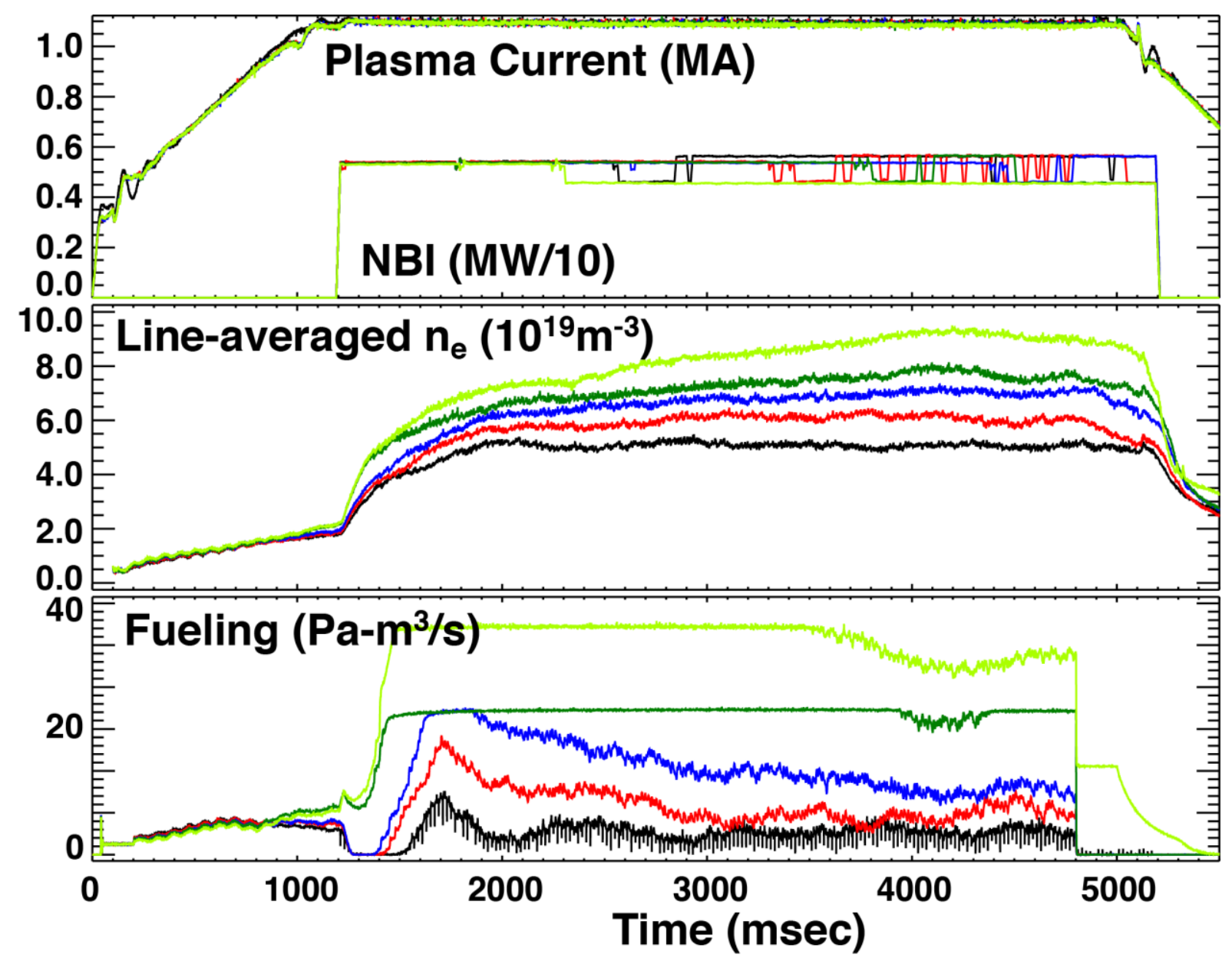

A. Leonard, Fig 1 


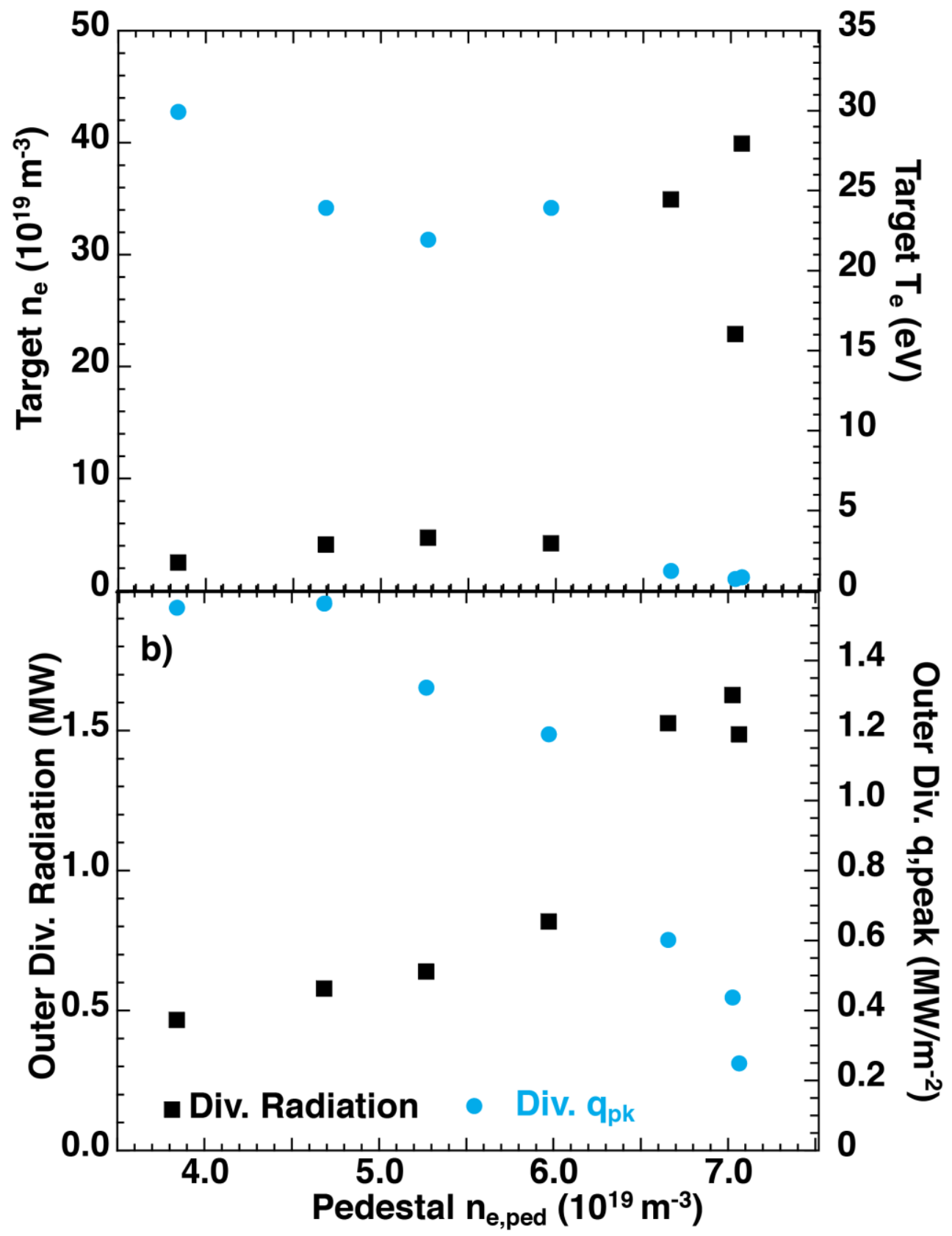

A. Leonard, Fig 2 

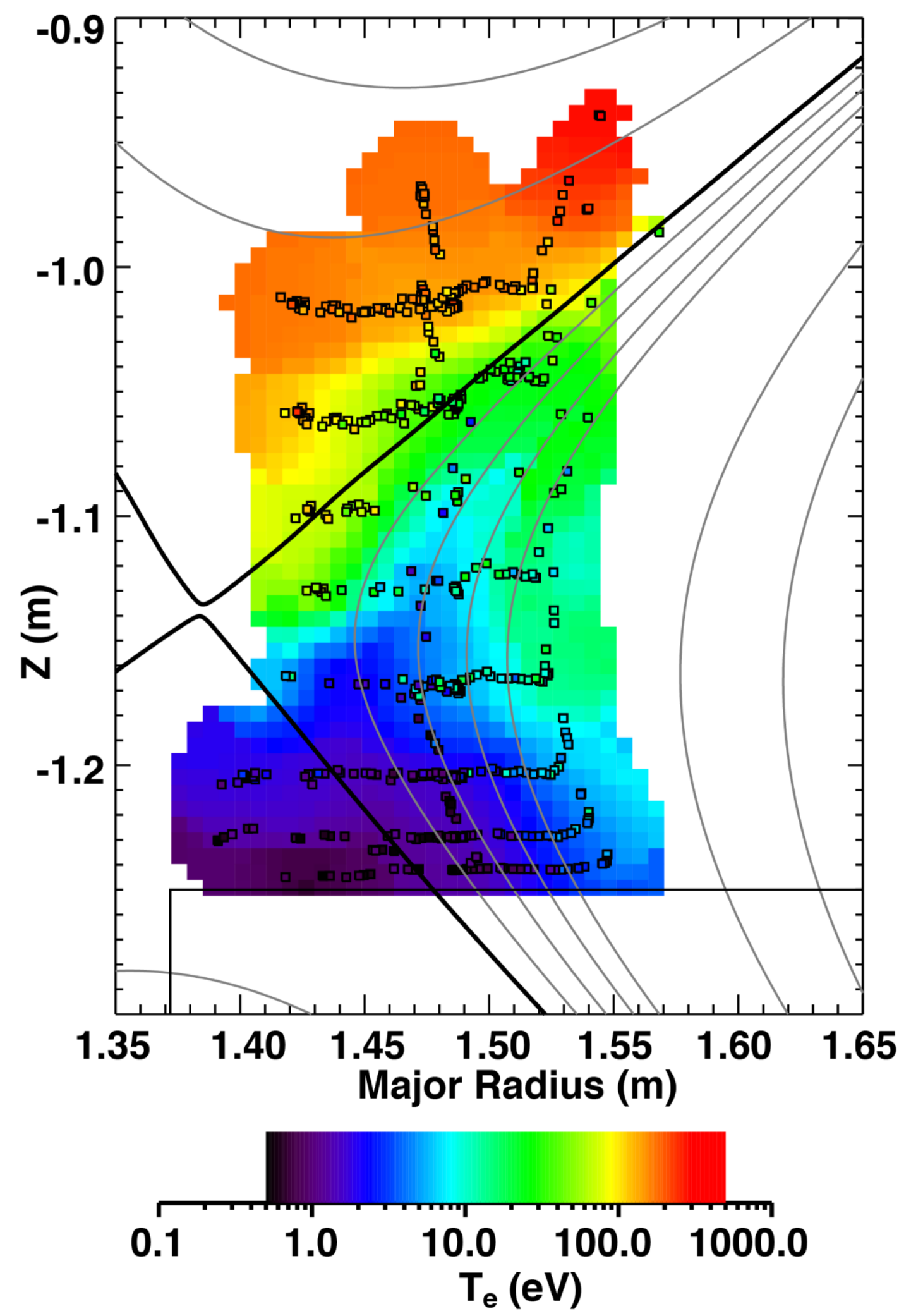

A. Leonard, Fig 3 

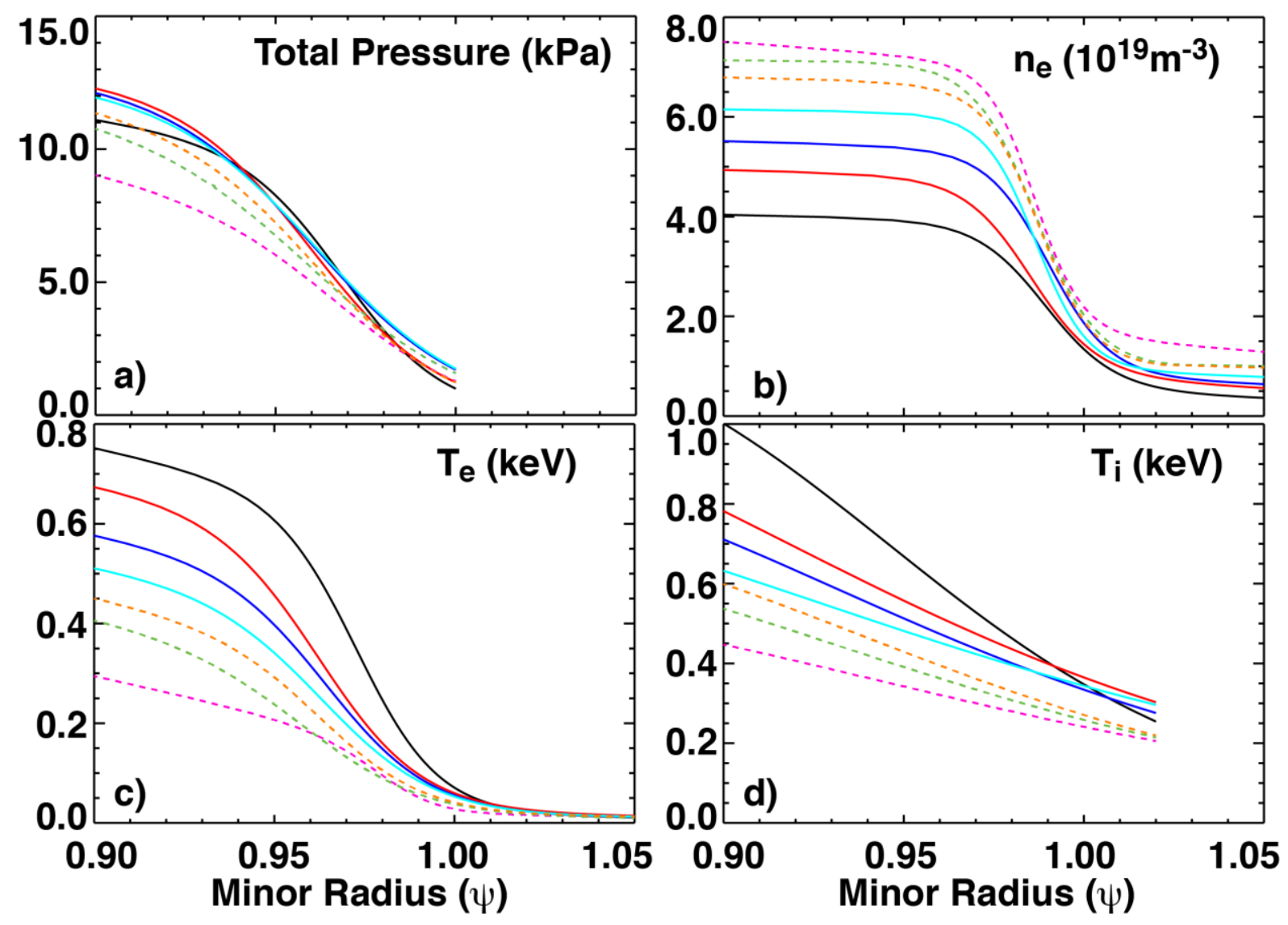

A. Leonard, Fig 4 


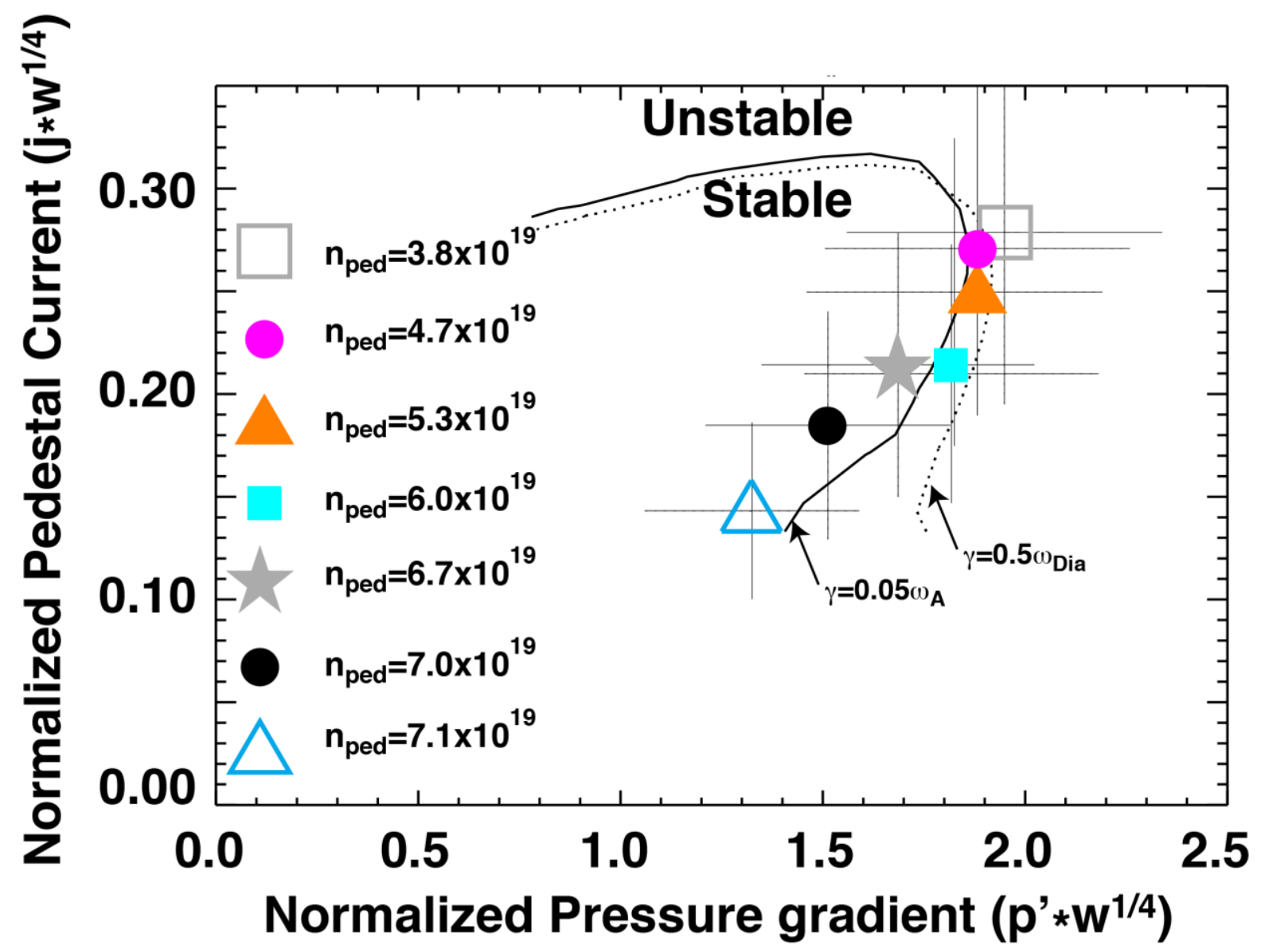

A. Leonard, Fig 5 


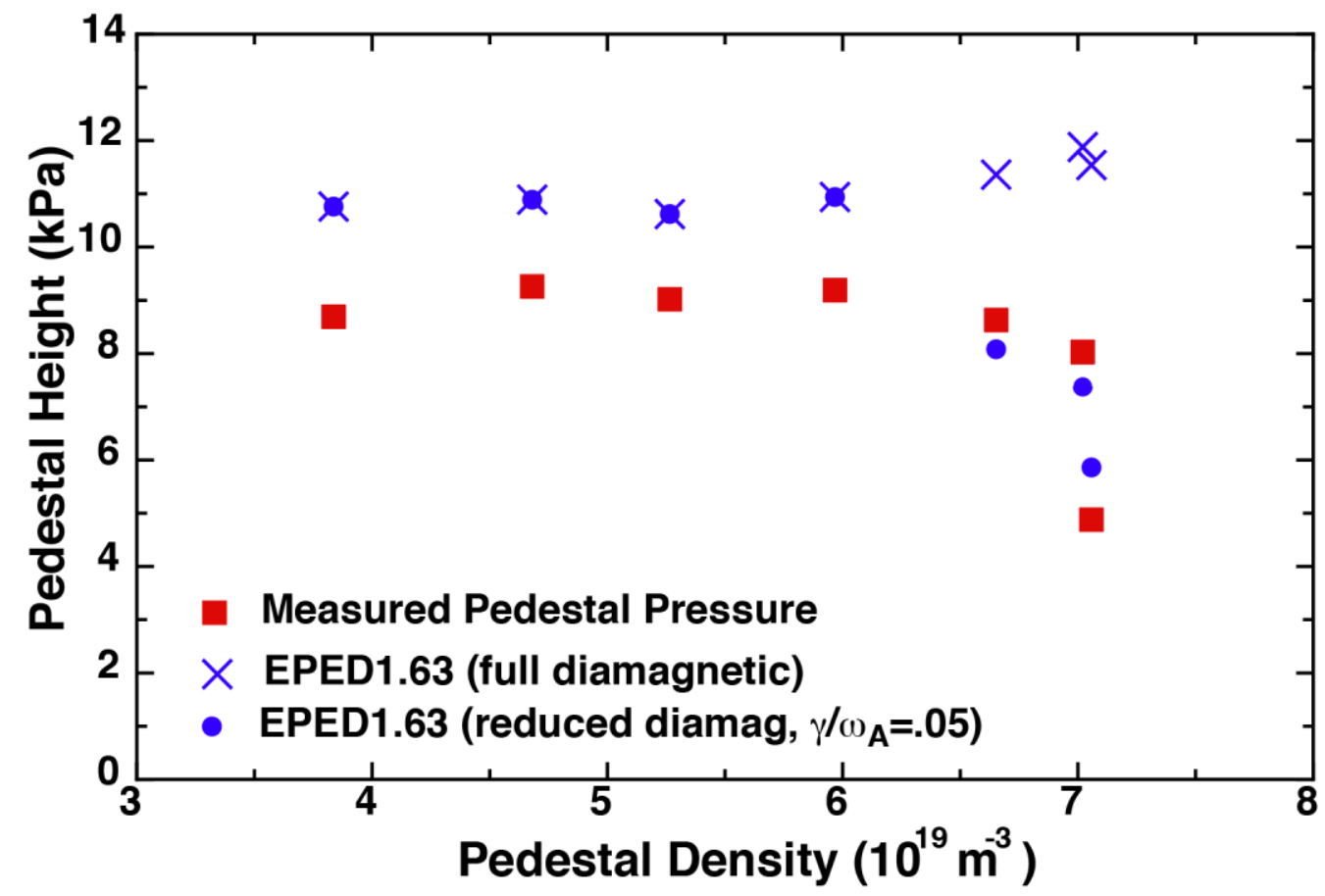

A. Leonard, Fig 6 